\title{
Precise Measurement of Cat Patellofemoral Joint Surface Geometry With Multistation Digital Photogrammetry
}

J. L. Ronsky

emall: Jlronsky@ucalgary ca

S. K. Boyd

Department of Mechanical Engineering.

\section{D. Lichti}

\section{A. Chapman}

Department of Geomatıcs Engineerıng

\author{
K. Šalkauskas \\ Department of Mathematics and Slatıstıcs.
}

University of Calgary. 2500 University Drive, N.W. Calgary, T2P 1N4 Canada
Three-dimensional joint models are important tools for investigating mechanisms related to normal and pathological joints. Often these models necessitate accurate three-dimensional joint surface geometric data so that reliable model results can be obtained; however, in models based on small joints, this is often problematic due to limitations of the present techniques. These limitations include insufficient measurement precision, the requirement of contact for the measurement process, and lack of entire joint description. This study presents a new non-contact method for precise determination of entire joint surfaces using multistation digital photogrammetry ( $M D P G$ ) and is demonstrated by determining the cartilage and subchondral bone surfaces of the cat patellofemoral $(P F)$ joint. The digital camera-lens setup was precisely calibrated using 16 photographs arranged to achieve highly convergent geometry to estimate interior and distortion parameters of the camera-lens setup. Subsequently, six photographs of each joint surface were then acquired for surface measurement. The digital images were directly imported to a computer and newly introduced semi-automatic computer algorithms were used to precisely determine the image coordinates. Finally, a rigorous mathematical procedure named the bundle adjustment was used to determine the three-dimensional coordinates of the joint surfaces and to estimate the precision of the coordinates. These estimations were validated by comparing the MDPG measurements of a cylinder and plane to an analytical model. The joint surfaces were successfully measured using the MDPG method with mean precision estimates in the least favorable coordinate direction being $10.3 \mu \mathrm{m}$ for subchondral bone and $17.9 \mu \mathrm{m}$ for cartilage. The difference in measurement precision for bone and cartilage primarily reflects differences in the translucent properties of the surfaces.

\section{Introduction}

Biomechanical factors are speculated to play a key role in the mechanisms associated with diarthrodial joint disorders, such as the development of osteoarthritis (OA). Experimental approaches to investigate relations between biomechanical factors and degenerative joint diseases have been limited. Experimental measures may be made on human diarthrodial joints in vitro (e.g., Blankevoort et al., 1988), and in some cases in vivo (e.g., Ronsky, 1994). However, animals are often used for joint studies because the experiments can be easily controlled (Bray et al., 1992; Herzog et al., 1993; Korvick et al., 1994; Boyd and Ronsky, 1998) and invasive procedures can be used such as surgical transection to artificially modify structural integrity of the joint (e.g., Pond and Nuki, 1973; Ronsky et al., 1995).

Analytical joint models implemented with these experimental measures provide a powerful approach to analysis of joint contact mechanics. An analytical model of the joint can be constructed for further mechanical analysis, and to allow systematic investigation of the effect of individual model parameters. An analytical model of a joint requires accurate geometric measures of the joint surface, including cartilage and subchondral bone, so mechanical properties that are related to joint disorders can be studied such as cartilage-cartilage contact, cartilage thickness, and joint surface curvatures. Surface geometry accuracy

Contributed by the Bloengineering Division for publication in the Jinte VAL of Biomechanical EngineEring. Manuscript received by the Bir engineerng Division January 8, 1998; revised manuscript recelved December I I'ر4k issuciate Technical Editor' R. Vanderbv. Jr. is problematic in small animal joints because the cartilage thickness may be within the error bounds of the surface measurement technique. For example, the cartilage thickness in the cat in the patellofemoral (PF) joint is as thin as $100 \mu \mathrm{m}$, which requires very precise measurements.

Several methods have been presented in the biomechanics literature for obtaining joint surface geometry. These include mechanical methods that require contact with the joint surface such as plastic moldings (Seedhom et al, 1972), plaster moldings measured by a coordinate measuring machine (Scherrer and Hillberry, 1979), a measuring pin attached to a dial gage (Wismans et al., 1980), and specimen slicing (Shiba et al., 1988 ). Non-contact optical methods that have been applied include Moiré contourography (e.g., Wijk, 1980), close-range photogrammetry (Ghosh, 1983), analytical stereophotogrammetry (SPG) (e.g., Huiskes et al., 1985; Ateshian et al., 1994), and magnetic resonance imaging (MRI) (e.g., Ronsky, 1994).

None of these techniques are satisfactory for three-dimensional reconstruction of small joints. Specimen slicing is difficult to perform on small joints and necessitates destroying the joint. This precludes the possibility of performing histological studies on the same joint at a later time. Contact techniques are problematic for measuring surfaces of tissues such as cartilage because associated tissue deformation may compromise the accuracy. Furthermore, maintaining constant hydration (Ronsky et al., 1997) is difficult during these time-consuming processes and may result in inaccuracy due to changes in tissue volume and subsequent tissue surface. MRI has limited resolution for small joints due to the sparsity of data between slices and limita- 
tion of total number of data points available from the image. SPG is the most promising technique because it is non-contact, data acquisition time is short thus reducing hydration error effects, and a sufficient number of surface measurements can be acquired so that a reliable three-dimensional reconstruction of the joint is achievable.

The SPG measurement procedure has been well described by Huiskes et al. (1985) and Areshian et al. (1991). Summarizing, it can be divided into three steps. First, two spatially separated photographs are taken of the joint surface mounted in a calibration frame while a grid is projected onto the surface. Second, the photographs are developed and the grid points and calibration frame targets are digitized from the photographic negatuves. Third, these digitized points are reconstructed using a re-section and intersection mathematical procedure to obtain three-dimensional surface coordinates.

Although SPG is a valuable tool for joint surface measurement, there are some limitations of the technique. First, the measurement precision is not sufficient for small joints where the cartilage thickness may be as low as $100 \mu \mathrm{m}$ as in the cat patellofemoral (PF) joint. The reported SPG measurement precision was $90 \mu \mathrm{m}$ (Ateshian et al., 1991) and $25 \mu \mathrm{m}$ (Ateshian et al., 1992) based on calibration measurements in ideal photogrammetric conditions, which likely do not represent actual experimental measurement conditions. Second, the use of two photographs for the three-dimensional reconstruction limits the ability to measure surfaces of joints that wrap (i.e., distal femur) because the surface region measured must be visible in both photographs. This problem can be overcome by performing multiple SPG reconstructions of different regions of the joint and then orienting the multiple surface measurements into the same coordinate system; however, this process is cumbersome. Third, an averaged three-dimensional surface point reconstruction error has been determined in one study (Ateshian et al., 1991), and the same measurement accuracy is assumed to be equal for other SPG measurements with similar setups (e.g., Kwak et al., 1997; Soslowsky et al., 1992). In fact, every reconstructed point on a surface has a different associated measurement error, and the overall average measurement error varies with different photogrammetric conditions (Fraser, 1982). Reliable error estimates are important for developing mathematical surface models that accurately represent the true joint surface. Fourth, the SPG method contunues to use a reconstruction process involving large format cameras, film developing, and hand digitization of photographic targets (e.g., Kwak et al., 1997), which, although practical during the development of the procedure, could be replaced by more efficient processes.

Multistation digital photogrammetry (MDPG) is an altemative form of photogrammetry that has been used in other scientific fields, for example, measurıng machine deformations ( $\mathrm{Co}-$ sandier and Chapman, 1992). It differs significantly from SPG and holds several advantages. MDPG is defined as the measurement of an object where all of the surface regions of interest are imaged on three or more spatially separated photographs (Granshaw, 1980). The sum of the individual targets viewed from each camera station in a photogrammetric session is defined as the photogrammetric network that is the basis of the three-dimensional reconstruction. The use of multiple images allows highly accurate determination of camera-lens parameters due to highly convergent camera angles ( $90 \mathrm{deg}$ ), which subsequently enhances the accuracy and reliabılıty of photogrammetric measurements (Granshaw, 1980; Fraser, 1980), and results in more homogeneous coordinate precision estimates (Fraser, 1984). The increased flexibility in camera positions permits complete coverage of highly curved joints because not all surtace regions of interest must be viewed from every camera position. The digital format camera eliminates film processing, reduces errors due to the emulsion technique or film flatness, and does not necessitate fiducial marks because these are intrinSIC in the charged-couple device (CCD). Additionally, two- dimensional point coordinate determination from the grid can be performed more efficiently and accurately using computer algorithms. The multiple observations from different camera positions are optimally reconstructed into their three-dimensional surface coordinates using a mathematical procedure called a bundle adjustment (Atkinson, 1996; Lichti et al., 1997).

The bundle adjustment method is a simultaneous solution, using least-squares optimization, of all the image rays mathematically defined by the collinearity equations in a photogrammetric network (Atkinson, 1996):

$$
\begin{aligned}
& x_{i,}-x_{p}+\delta x+\xi x \\
& =f \frac{\left(X_{1}-X_{c, j}\right) R_{11, j}+\left(Y_{\imath}-Y_{c, \jmath}\right) R_{12, j}+\left(Z_{1}-Z_{c, j}\right) R_{13, j}}{\left(X_{1}-X_{c, v}\right) R_{31, j}+\left(Y_{1}-Y_{c, j}\right) R_{32, j}+\left(Z_{i}-Z_{c, j}\right) R_{33, j}}
\end{aligned}
$$

$$
\begin{aligned}
y_{1,}- & y_{p}+\delta y+\xi y \\
& =f \frac{\left(X_{t}-X_{c,}\right) R_{21, j}+\left(Y_{1}-Y_{c \jmath}\right) R_{22, j}+\left(Z_{t}-Z_{c, j}\right) R_{23, J}}{\left(X_{t}-X_{c \jmath}\right) R_{31, j}+\left(Y_{i}-Y_{c \jmath}\right) R_{32, J}+\left(Z_{1}-Z_{c, J}\right) R_{33, j}}
\end{aligned}
$$

The bundle adjustment method is a general term that encompasses two specific forms of the method. These two forms are referred to as a self-calibrating bundle adjustment or a bundle adjustment depending on the number of parameters being solved in the simultaneous solution. The self-calibrating bundle adjustment refers to the simultaneous solution of parameters defining the exterior orientation of the camera positions $\left(X_{c_{v}}, Y_{c_{v}}, Z_{c_{v}}\right.$, $\left.R_{s, j}\right)$, interior orientation of the camera $\left(x_{p}, y_{p}, f\right)$ and distortion parameters in a photogrammetric setup. Alternatively, the bundle adjustment solves fewer parameters because it treats the interior $\left(x_{p}, y_{p}, f\right)$ and distortion parameters as known, and solves for only the exterior $\left(X_{c_{j}}, Y_{c j}, Z_{c, j}, R_{s, j}\right)$ parameters simultaneously with the object points $\left(X_{i}, Y_{1}, Z_{i}\right)$. Both bundle adjustment methods use image points measurements $\left(x_{1,}, y_{1 j}\right)$ as input to the least-squares optimization of Eqs. (1) and (2). A rigorous description of the implementation of the bundle adjustment is provided by Atkinson (1996).

The purpose of this study is to incorporate significant advancements in the field of photogrammetry into a photogrammetric technique utilizing MDPG that is capable of producing highly accurate joint surface measurements and is flexible for different measuring conditions encountered in biomechanics research. The technique is first tested by measuring known die shapes and comparing the results to an analytical model of those shapes. Then the technique is used to measure the cartilage and subchondral bone of the femur and patella of the cat PF joint.

\section{Experimental Methods}

The first part of the methods section describes the preparation of the measurement specimens. The second part describes the standard MDPG protocol used to measure the specimen surfaces in four stages. In the first stage, a series of photographs of a calibration frame is taken for camera-lens calibration. Second, a series of photographs for each type of specimen surface is obtained for surface measurements. Third, the two-dimensional coordinates of image targets in the digital photographs are determined using custom edge detection and pattern fitting routines. Fourth, these two-dimensional points from the calibration and the surface series of photographs are reconstructed using the bundle adjustment method to determine camera-lens calibration parameters and threedimensional surface coordinates, respectively.

Specimen Surfaces. Two die shapes were used to validate the MDPG measurement precision estimates from the bundle adjustment. Because the shapes of dies were known a priori, 


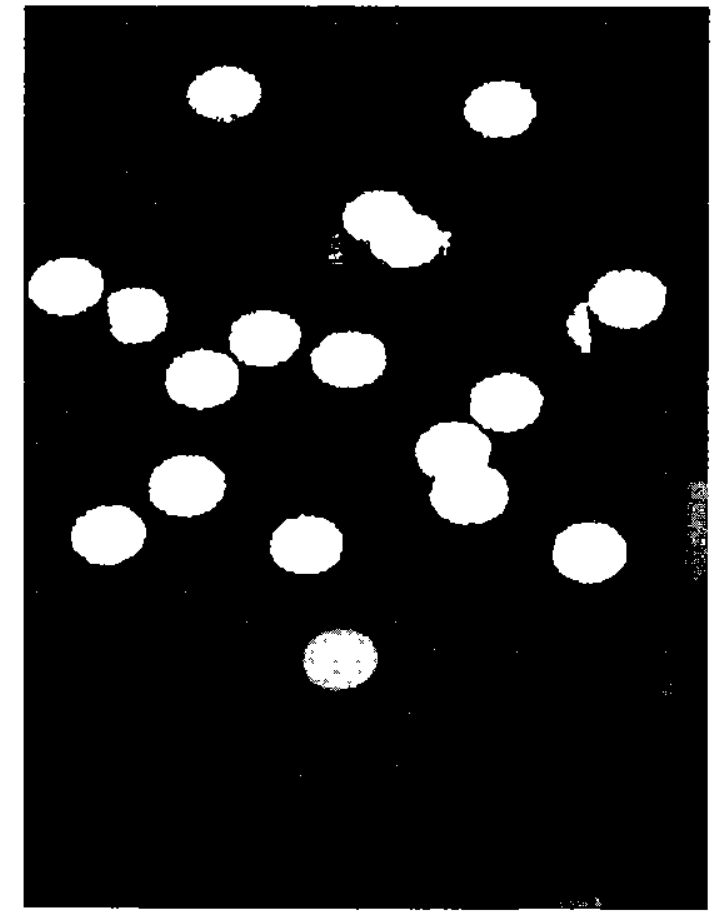

Fig. 1 The target field used for calibration of the camera-lens system consisting of 19 control posts. Base diameter is $50 \mathrm{~mm}$.

these precision estimates could be directly compared to the error of the three-dimensional points coordinates fit to an analytical model of the die shapes. Surface measurements of a cylinder $(6.350 \mathrm{~mm}$ radius, S.D. $=10.2 \mu \mathrm{m})$ and a plane ( finely polished with a $2 \mu \mathrm{m}$ grit paper) were obtained using the standard experimental protocol. The radius of the cylinder was chosen so that its curvature was similar to the maximum curvature in the cat $P F$ joint.

Three mature outbred male cats $(4.5 \pm 0.5 \mathrm{~kg})$ were used for the joint measurements. Approval for this study was obtained from the University Animal Care Committee. The patella and femur were freshly harvested and mounted in individual frames so the cartilage surfaces could be measured. After being photographed, the joint surfaces in their frames were immersed in a solution of bleach for three hours to dissolve the cartilage and expose the underlying subchondral bone for subsequent measurements. This protocol, adapted from Ateshian et al. (1991), gently removes the cartilage layer without disturbing the position of the specimen in its frame.

MDPG Method: Photographic Acquisition for Calibration. The camera-lens configuration used for the photogrammetric data collection was calibrated to obtain optimal reconstruction accuracy. This was achieved using a highly redundant imaging network of an isotropically distributed target field to determine intemal geometric and distortion parameters that are unique for every photogrammetric setup (Fraser et al., 1995). These parameters are very sensitive to changes in the lens focal setting, aperture ( $f / s t o p)$, and interface to the camera body (i.e., detachment and re-attachment of the lens will effect interior geometries) and therefore calibration was performed before every photogrammetric experiment.

A Kodak DCS 420 digital camera (Eastman Kodak Company, Rochester, NY) with a CCD sensor resolution of 1524 $\times 1012$ pixels ( $9 \mu \mathrm{m}$ sensor element spacing in both directions) was used. The camera was fitted with a Nikkor $35-135 \mathrm{~mm}$ f/ 3.5-4.5 macro-focusing zoom lens (Nikon, Nippon Kogaku KK, Japan). A long focal length lens contributes to the target positioning accuracy (Fraser, 1984).

Two styles of calibration frames were used for this study. The first was a prototype and was used with specimens one and two. It consisted of a target field of 20 steel posts (dia $5 \mathrm{~mm}$ ) rigidly mounted in an $80 \mathrm{~mm}$ circular pattern. The second frame, used for the third specimen, was an improved design because the target field was more isotropically distributed, rather than targets being located around the periphery. It consisted of a target field of 19 steel posts (dia $5 \mathrm{~mm}$ ) rigidly mounted in concentric circles on a 50-mm-diam base (Fig. 1). For both frames the height variation between posts was maximally 14 .mm. The target tops were painted flat white, and the remainder was painted black for high contrast. The positions of each target post were measured with a coordinate measuring machine (CMM) machine ( $<2 \mu \mathrm{m}$ accuracy) (Model FJ805, Mitutoyo MTI Corporation, Japan).

The target imaging of the calibration frame included two photos (oriented landscape and portrait) from eight equally spaced camera positions around the calibration target field ( 16 photos total, each pair spaced radially by $45 \mathrm{deg}$ ) from a distance of approximatély $300 \mathrm{~mm}$. The camera positions optical axes formed an angle of approximately $45 \mathrm{deg}$ with the vertical axis of the calibration field, thus a $90 \mathrm{deg}$ convergence angle was obtained between opposing pairs of photographs. Rather than move the camera around the frame for eight equally spaced camera positions, it remained stationary on the tripod and the calibration frame was rotated eight times. The lens was set at an aperture stop of $f / 22$ to maximize the depth of field. The high aperture stop setting necessitated the use of a lamp and a shutter speed of one second to expose the images properly. The lens focal ring was locked in position to prevent accidental disturbance for the duration of surface geometry data acquisition. The 16 photos of the targets formed the calibration imaging network from which precise camera-lens interior geometries and any distortions in the camera-lens setup could be determined using the self-calibrating bundle adjustment.

MDPG Method: Photographic Acquisition for Surface Measurement. The femur and patella of specimens one and two were mounted in the center of the $80 \mathrm{~mm}$ circular arrangement of target posts of the prototype frame that was also used for the calibration. The femur and patella from specimen three, and the cylinder and plane specimens, were mounted in specialized rings consisting of eight equally spaced posts (dia $33 \mathrm{~mm}$ ) rigidly mounted around the specimen (Fig. 2). Similar to the calibration target field, the post tops were painted white and the remainder painted black. The specimen ring provides a reference for the object space coordinate system during the photo-

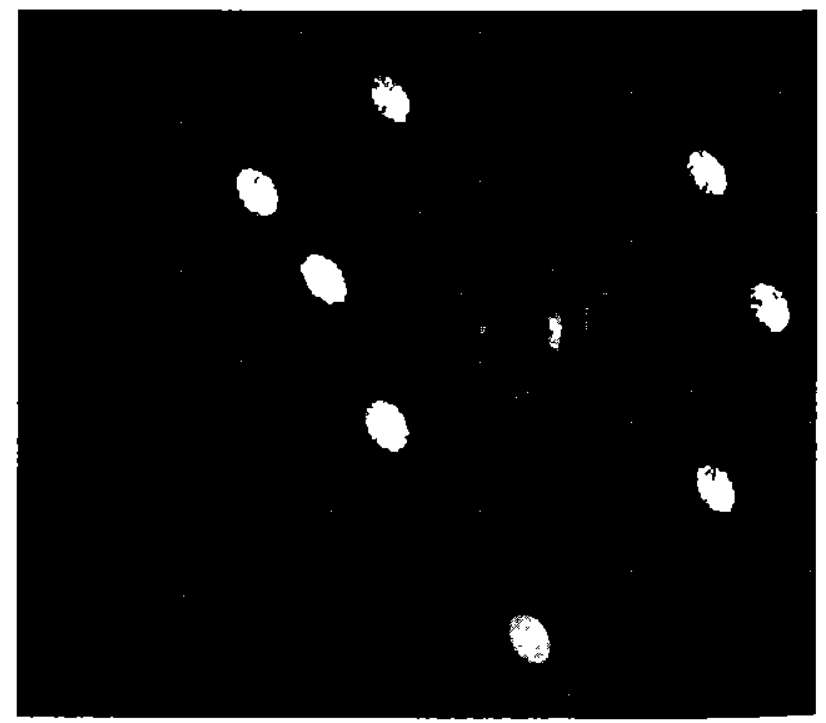

Fig. 2 A sample photographic image of the specimen surface (diatal femur with cartlage) and the specimen ring. The eight posts on the specimen ring provide reference points for three-dimensional object space. 


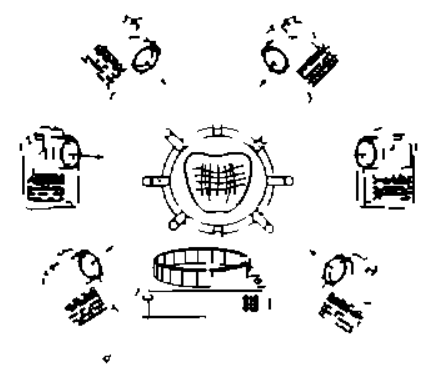

Fig. 3 Multistation digıtal photogrammetric setup. A slide projector projects a grid pattern onto the specimen surface. The specimen ring is mounted on the specimen. One camera takes photographs of the object from six positions. The computer used for two-dimensional digitization and three-dimensional reconstruction is not shown.

gramınetric reconstruction. It was designed to fit as closely around the specimen surface as possible (i.e., Fig. 2) so that the magnification of the surfaces was maximized in the photographs

A standard slide projector (Kodak Model $760 \mathrm{H}$. Eastman Kodak Company, Rochester, NY) projected a uniform rectangular grid onto the specimen surface. The grid spacing was constant across the specimen surface $(0.75 \mathrm{~mm})$ and the line thickness was approximately $0.20 \mathrm{~mm}$. The purpose of the grıd was to provide reference marks on the surfaces, which are otherwise featureless (Huiskes et al., 1985). With the camera stabilized using a tripod, six photos equally spaced in a radial pattern around the specimen (Fig. 3 ) were manually taken in sequence from a distance of $300 \mathrm{~mm}$. A series of six photos took approximately five minutes to collect for each surface being measured. After each photo session, the images were viewed on a computer screen to ensure adequate exposure. If necessary, the shutter speed was adjusted, but never the aperature (depth of field) because it would likely alter the lens distortion. In the case of the femur of specimen three the femoral epicondyles were measured in addition to the femoral groove. Because the grid could not be projected onto the entire joint surface during one data acquisition, the grid was repositioned and a second data acquisition was performed. Both sets of measurements were measured in the same reference coordinate system provided by the specimen ring.

MDPG Method: Digitizing Images. The accuracy of the two-dimensional coordinates determined for the image targets in the digital photographs directly affects the three-dimensional reconstruction accuracy. To provide maxımal accuracy, edge detection and pattern fitting techniques were applied to the digital images of the calıbration target field and the specimen surfaces. The image coordinates had to be determined for two basic target types: control post targets (e.g., Fig. 1) and grid intersection targets on specimen surfaces (e.g., Fig. 2).

Control Posts. The contrast of the white circular top and the black background makes edge detection possible for determining the two-dimensional image coordinates of the post centers. Utilizing the properly that a flat circular target appears as an ellipse from all oblique vicws, an edge detection algorithm was adapted from an exisung algorithm (Cosandier and Chapman, 1992) that found all the edge points to sub-pixel accuracy (Tabatabai and Mitchell, 1984). The program (Mallab, v4.2, Mathworks, Inc., Natick, MA) finds the center of gravity (pixels are weighted according to aray-scale levels), divides the control post into four regions. and applies the one-dimensionat edge detector in each region (Fig. 4). After all the edges have beell determined, they are fit with an analytical model of an ellipse using the least-squares method. The center of the ellipse is the two-dimensional image position of the control post, and the rins value of the analytical ellipse fit to the edge points gives a measure of the quality of the fit. The program output consists of the following for each target: an identification number, the camera position number from which the target was digitized, the two-dimensional target coordinates on the photograph, and the rms fit of the target coordinates.

Grid Intersections. A semi-automatic digitizing program was developed for determining the precise location of the grid intersection points based on simple pattern fitting (Matlab, v4.2, Mathworks, Inc., Natick, MA). This program fit polynomials to the projected horizontal and vertical grid lines and then determined their intersections to get two-dimensional image coordinates of the grid intersections. Edge detection was not used to determine grid intersections because the cross-sectional pixel intensity of a line was defined by too few pixels $(<10)$ to determine the line edges reliably.

The grid intersection program was also semi-automated. First, the user chooses points along a horizontal or vertical line with a mouse, and the algorithm determines the exact line center at each point (Fig. 5( $a)$ ). This is done for all the horizontal and vertical lines on the digital photograph. The exact determination of the line center is performed automatically for each individual sample point as follows: From the sample point, the algorithm reads the gray-scale pixel intensity 10 pixels to the left and right ( 20 pixels rotal) for a vertical line (Fig. $5(b)$ ), or 10 pixels above and below for a horizontal line. The size of the cross section can be varied and a size of 20 was chosen because line width was typically between 5 to 10 pixels. A total sample width of 20 pixels ensured that the middle of the line is included in the sample if the operator does not put the mouse cursor exactly on the line center, but that neighboring lines will not be accidentally included. After the cross section has been determined, the algorithm finds the darkest grey-scale pixel intensity ( $0=$ black, $1=$ white) that indicates the precise center of the line (Fig. $5(c)$ ). The line center points that were calculated by the algorithm from the user-defined sample points describe the horizontal and vertical grid lines and were fit with fifth-order polynomials. The fifth-order polynomial was chosen because it proved most flexible at reliably reproducing the shape of the line with minimal oscillations. After the polynomials had been determined for each horizontal and vertical line, the grid intersections were calculated automatically by solving for the intersection of each horizontal and vertical polynomial (Fig. 6(a)). On occasions that a grid intersection determined automatically was not satisfactory upon visual inspection by the user, a new grid intersection could be manually defined by zooming the image and defining the intersection manually with a mouse. Unsatisfactory grid intersection determination was most common near the edges of the joint surface where the polynomial was unable to follow the grid line due to lack of samples near the ends. After a satisfactory grid is obtained, the points are identified by row and column with a mouse so that the same point can be identified in each camera view (Fig. $6(b)$ ). The program output consists of the following for each target: an identification number, the camera position number from which the target was digitized, and the two-dimensional target position on the photograph.

MDPG Method: Bundle Adjustments. The distortion model that was applied in the bundle adjustments (Fig. 7) in this MDPG study included parameters to model radial distortion $\left(k_{1}, k_{2}, k_{3}\right)$, decentering distortion $\left(p_{1}, p_{2}\right)$, differential scaling between vertical and horizontal pixel spacing on the CCD chip sensor surface $\left(a_{1}\right)$, and shearing distortion on the CCD chip $\left(a_{2}\right)$ (Fraser et al., 1995; Lichti et al., 1997):

$$
\begin{aligned}
& \delta x=\left(k_{1} r^{2}+k_{2} r^{4}+k_{3} r^{6}\right) \bar{x}+p_{1}\left(r^{2}+2 \bar{x}^{2}\right) \\
&+2 p_{2} \overline{x y}+a_{1} \bar{x}+a_{2} \bar{y} \\
& \delta y=\left(k_{1} r^{2}+k_{2} r^{4}+k_{3} r^{6}\right) \bar{y}+2 p_{1} \overline{x y}+p_{2}\left(r^{2}+2 \bar{y}^{2}\right)
\end{aligned}
$$

The self-calibrating bundle adjustment, which achieves the 
(A)

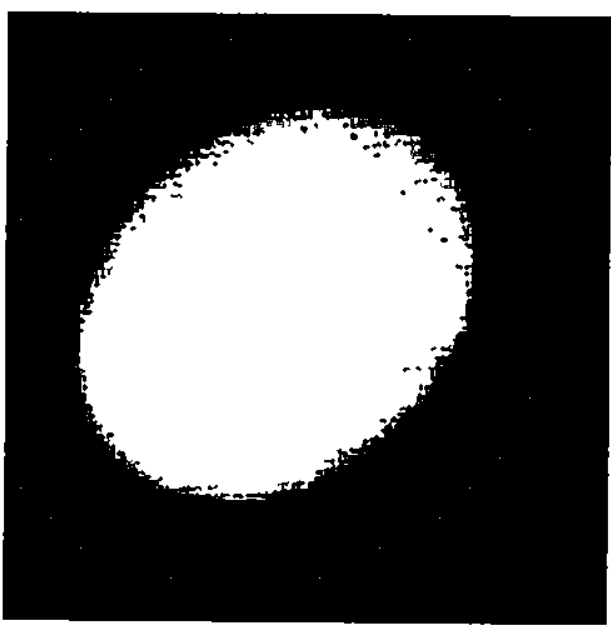

(B)

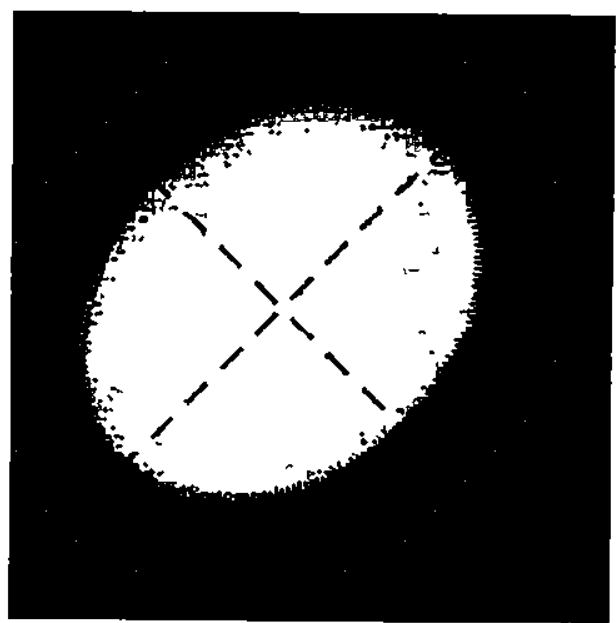

Fig. 4 A close-up of the white top of one of the control posts $(A)$ cropped by the user from the original photogrammetric image. The same image after edge detection has been applied (B). The algorithm finds the gray-scale intensity center-of-mass and divides the cylinder into four regions (dashed lines divide four regions for diagramatic purposes). One-dimensional edge detection is systematically applied to the four regions to find the control post edges (+). An ellipse is fit to the edge points to find the two-dimensional image position of the post centre.

greatest possible positioning accuracy in a photogrammetric reconstruction (Fraser, 1982), was used to determine interior and distortion parameters and thus calibrate the camera-lens system. Resolving these parameters, especially the higher order distortion terms, requires a well-designed photogrammetric network (Lichti et al., 1997). The network design formed in this study included features of a well-designed network, which consisted of the digitized two-dimensional positions of the isotropi-

(A)
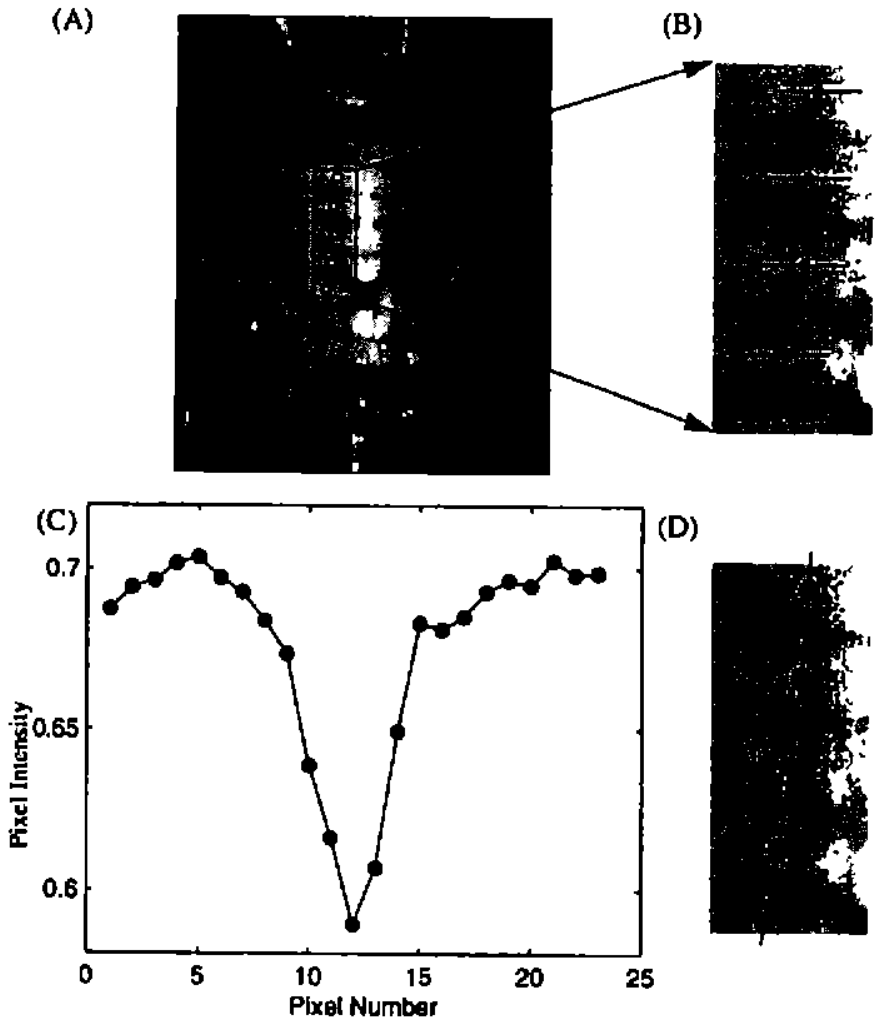

(D)

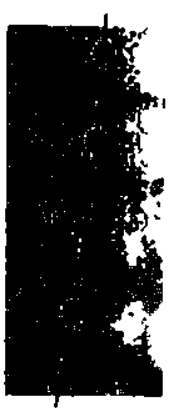

Fig. 5 (A) Sample of a digital image of a cat patella articular cartilage surface with a projected grid pattem. The hollow clrcles indicate user input sempling regions. (B) Close-up of a portion of the grid pattern. The dark horizontal lines iliustrate a typlcal sample cross section of the image pixels. (C) The pixel intensities of one sample cross section of the grid line. After fitting a spline to the cross section, the darkest subplxel value (l.e., minimum pixel intensity) ls taken as the center of the Ine. (D) The precise center of the line is determined at several sample points along the line and a fifth-order polynomial is fit to describe the line. cally distributed posts on the calibration frame from the 16 highly convergent (i.e., 90 deg convergence angle) photographs that were nominally orthogonal (i.e., camera rotated $90 \mathrm{deg}$ for two exposures at each camera position). From this photogrammetric network, a solution of the self-calibrating bundle adjustment was determined. Because the convergence of the solution is sensitive to initial estimates of the exterior and interior camera parameters, it was convenient to run it without distortion parameters for the first time. The estimated interior and exterior camera parameters from this solution were good initial estimates for further solutions that include distortion parameters. To determine a final solution including distortion parameters, the selfcalibrating bundle adjustment was repeatedly run with different combinations of distortion parameters included. Statistical tests were performed (Förstner, 1985; Fraser, 1980; Lichti, 1996; Lichti et al., 1997) to determine which distortion parameters are significant and should be included in the final self-calibrating bundle adjustment. Statistical methods were also used to find gross errors due to observational mistakes (Förstner, 1985). The calibration target coordinate position and precision was estimated in $x, y$, and $z$ for each calibration.
(A)

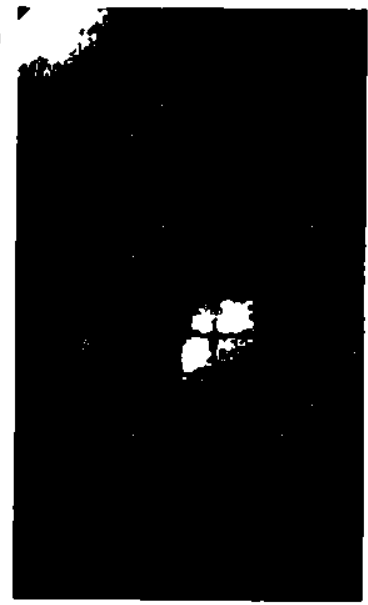

(B)

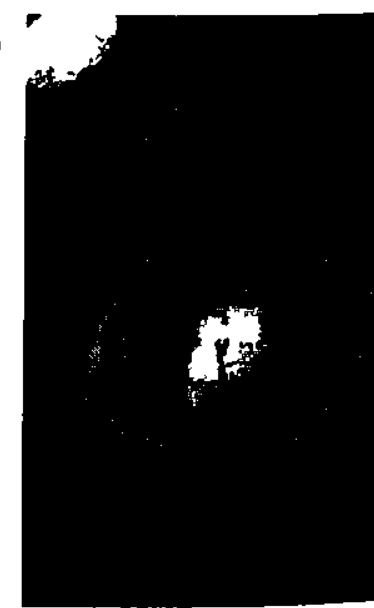

Flg. 6 (A) A close-up of a typlcal digital image of the joint Burface with the projected grid pattern. The horizontal and vertical lines have been determined and their intersections calculated. $(B)$ The polynomial intersection points corresponding to the grid intersections are labeled as columns and rows 80 that the seme grid point can be Identifled in each digital image. Poorty defined points are labeled 0,0 and the grid intersection is then defined manually after magnifying the image. 


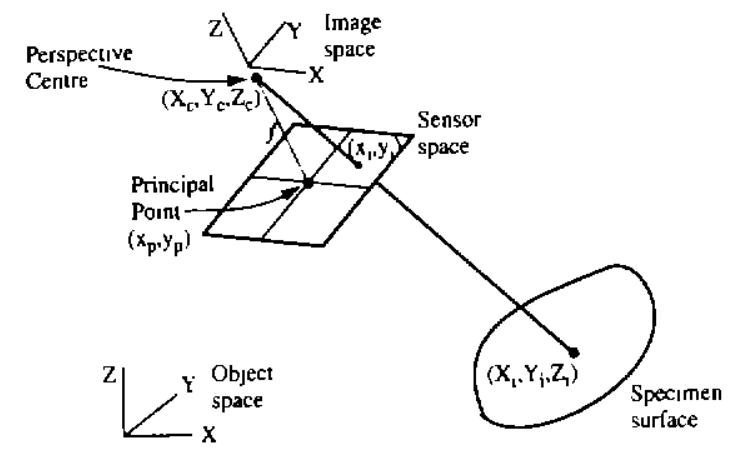

Fig 7 Schematic shows a representation of a camera imaging a speciFig. 7 scher onto a sensor (film or digital). The dark line from the permen surface onto a sensipiple of the spective center to the specimen surtace represents the prin in a photocollinearity condition. The coordinate systems of interest in a photogrammetric system are object space $\left(X_{1}, Y_{,}, Z_{1}\right)$, which is equivalent to grammetric space $\left(X_{c}, Y_{c}, Z_{c}\right)$ which defines a lab coordinate system, and mage space $\left(X_{c}, \gamma_{c 1} Z_{c}\right)$ which definestive the camera-lens interior geometry and has its origin at the perspecive center $\left(X, Y_{c}, Z_{c}\right)$. Sensor space is the sensor plane defined in image center $\left(x_{c}, \gamma_{1} z_{c}\right)$. space. The principal point $\left.x_{p}, y_{p}\right)$ is the plane and passing the intersection of a line perpendicular to the sensor plane and passing through the perspective center of the camera. The focal length $(f)$ defines the scale of the reconstructed image and must be determined very accurately.

After the self-calibrating bundle adjustment was completed, the estimated interior and distortion parameters of the cameralens system were used for the subsequent surface photogrammetric measurements Thus, the interior and distortion parameters for the camera-lens system were treated as known constants because they were previously solved using the self-calibranng bundle adjustment. The two-dimensional $\left(x_{t, 1}, y_{1,1}\right)$ positions of the targets (both posts and grid intersections) from the network of joint stiface photographs are used in the more simple bundle adjustment to find the simultaneous solution of the camera exterior parameters $\left(X_{6}, Y_{t, j}, Z_{c, t}, R_{t, j}\right)$, the precise three-dimensional surface measurements, and their as sociated estimated coordinate precision. The output of the bundle adjustment includes the three-dimensional spatial coordinates of each target (post top or grid intersection ), its identification number, the number of images in which it was viewed (normally six), and the estimated $\mathrm{sms}$ precision in the $x, y$, and $z$ directions.

Analysis of Surface Specimen Measurements. The threedimensional surface coordinates for the cylinder and the plane were fit using a least-squares optimization to an analytical model of a cylinder (five parameters) and a plane (three parameters), respectively. The root-mean square ( $\mathrm{rms}$ ) deviation of the three-dimensional surface coordinates from the analytical models is expected to be similar to the estımated precision for the surface coordinates determined using the MDPG method.

The three-dimensional coordinates from the cartilage and subchondral bone surfices of the thiee PF joints were inathematically modeled with the interpolatıng thin-plate spline (TPS) (Boyd et al., 1997) and a grid mesh was created to display the modeled joint surfaces.

\section{Results}

The 16 photograph calıbration networks that were used to calibrate the camera-lens selup for each session were able to reconstruct the three-dimensional calibration post positions to a precision of $2 \mu \mathrm{m}\left(\sigma_{1}=\sigma_{1}=\sigma=2 \mu \mathrm{m}\right)$ as estimated by the output of the self-calibrating bundle adjustments. This measurement precision represents the optımum possible precision for any subsequent surface measurements with the cilibrated camera-lens configurations. The only statistically significant distortion parameter, out of the possible seven, that was determined with the self-calıbration was the $a_{1}$ distortion parameter accounting for differentual scaling on the CCD chip.
Table 1 MDPG estimated mean measurement precision for the plane and cylinder determined from the MDPG method in each of the three coordinate directions

\begin{tabular}{|c|c|c|c|c|}
\hline \multirow{2}{*}{} & \multirow{2}{*}{\begin{tabular}{c}
$\#$ \\
\cline { 3 - 5 }
\end{tabular}} & \multicolumn{4}{|c|}{ mean $\sigma(\mu \mathrm{m})$} \\
\cline { 3 - 5 } & & $\sigma_{x}$ & $\sigma_{y}$ & $\sigma_{z}$ \\
\hline Plane & 183 & 2.1 & 2.1 & 3.1 \\
\hline Cylinder & 169 & 7.1 & 6.0 & 12.7 \\
\hline
\end{tabular}

Cylinder and Plane Specimens. The mean estimated precision of the individual surface coordinates for the cylinder and plane surfaces measured using the MDPG method were determined in the $x, y$, and $z$ directions (Table 1). The mean precision of the MDPG surface coordinate measurements vary depending on the surface being measured and the coordinate direction. The surface measurements are more precise for the plane than the cylinder.

The rms fit of the cylinder and plane measured surface points to an analytical model of a cylinder and plane were determined (Table 2). These results were compared to the estimated precision from MDPG (Table 1). For example, the MDPG estimated measurement precision for the plane surface, which is mainly attributed to measurements in the height ( $z$ coordinate direction) is $3.1 \mu \mathrm{m}$ ( Table 1 ) compared to the $1.1 \mu \mathrm{m}$ plane fit (Table 2). Similarly, the predicted measurement precision of the cylinder surface, which is attributed to both measurements in the $y$ and $z$ directions is $14.0 \mu \mathrm{m}$ (radial error is $\left(\sigma_{y}^{2}+\sigma_{z}^{2}\right)^{-05}=\left(6.0^{2}\right.$ $\left.+12.7^{2}\right)^{-05}=14.0 \mu \mathrm{m}$ ) compared to a $17.3 \mu \mathrm{m}$ cylinder fit eror. These independent comparisons for the plane and cylinder measurement error support the proposal that the mean measurement precision for the MDPG surface points determined by the bundle adjustment method provide a realistic estimate of the photogrammetric reconstruction error.

PF Joint Surfaces. The MDPG method was successfully employed to collect bone and cartilage surface measurements for the femur (Fig. 8) and patella (Fig. 9) in all three experimental specimens. The technique was refined from its initial use with specimen one, to the most surface coverage (including distal femoral epicondyles), the most dense grid projection $(0.75 \mathrm{~mm}$ spacing), and best measurement accuracy (as low as $10.5 \mu \mathrm{m}$ ) with specimen three. The improvement in surface coverage, grid density, and accuracy can be observed from the summary of results of the MDPG reconstruction with specimens one and two (Table 3), and specimen three (Table 4). Six images were successfully used to reconstruct every joint surface except for the patella bone surface of specimen one.

\section{Discussion}

MDPG was successfully used to obtain precise measurements of analytical surfaces and the cat PF joint. The MDPG method provides advantages of non-contact high precision measurement of small joints, improved efficiencies through the use of digital data processing, and a rigorous mathematical reconstruction procedure that estimates reconstruction error. Furthermore, the system is easily transportable, which facilitates measurement of joint surfaces at the actual experimental site and hence reduces

Table 2 The rms fit of observations of a cylinder and plane made by MDPG to their respective analytical models

\begin{tabular}{|c|c|c|c|}
\hline & $\begin{array}{c}\# \\
\text { Pts }\end{array}$ & $\begin{array}{c}\text { \# Est } \\
\text { Pare. }\end{array}$ & $\begin{array}{c}\text { RMS } \\
(\mu \mathrm{m})\end{array}$ \\
\hline \hline Plane & 183 & 3 & 1.1 \\
\hline Cylinder & 169 & 5 & 17.3 \\
\hline
\end{tabular}


(A)

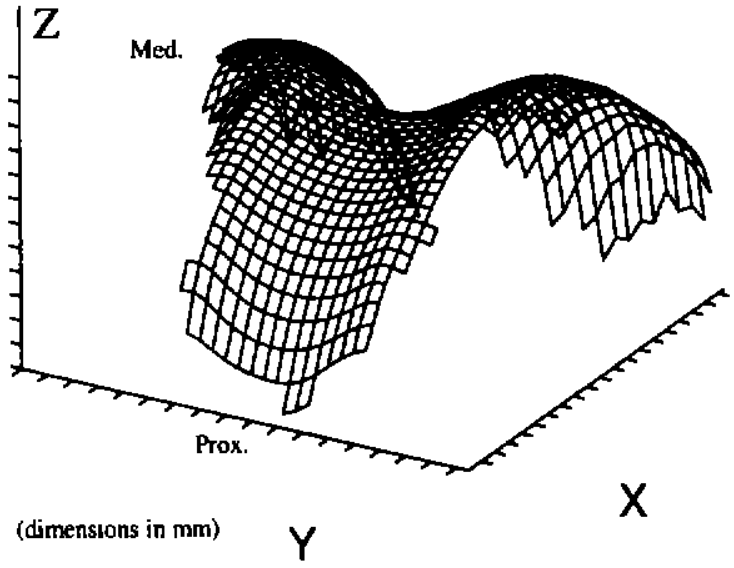

(B)

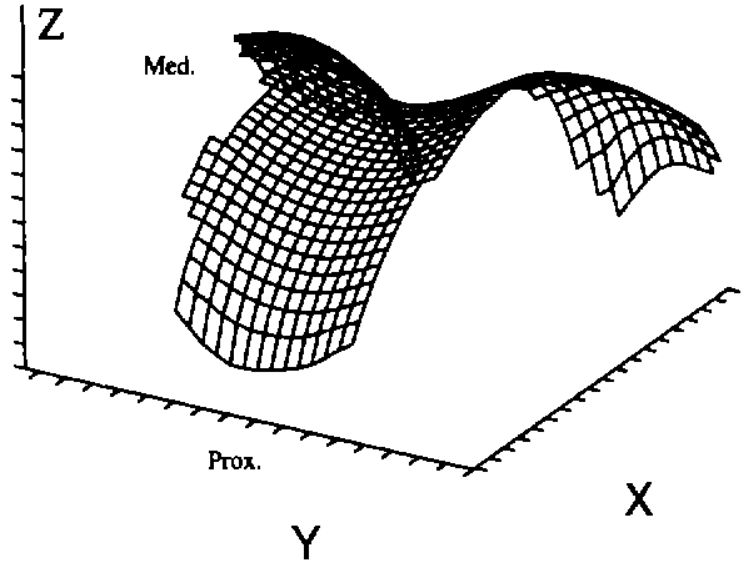

Fig. 8 Three-dimensional mesh grid measured on specimen 3 using MDPG data points and TPS modeling of the femur subchondral bone $(A)$ and cartilage surface $(B)$

(A)

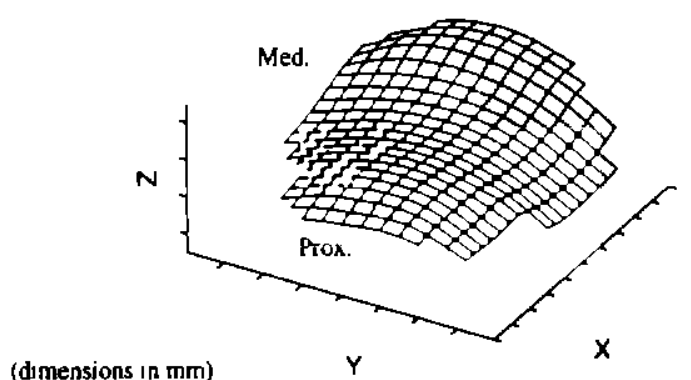

(B)

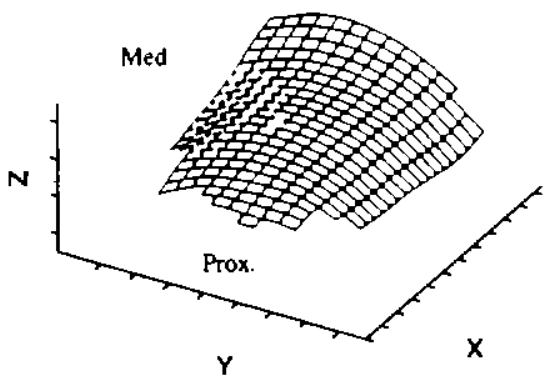

Fig. 9 Three-dimensional mesh grid measured on specimen 3 with MDPG data points and TPS modeling of the patella subchondral bone $(A)$ and cartilage surface $(B)$

the possibility of cartilage deformation due to lengthy exposure to air or storage treatment (i.e., freezing).

In this study, a grid pattern was projected onto the surfaces to provide identifiable marks on an otherwise featureless surface. In other studies it may not be desirable to project a grid. In this case, aiternative designs could be used such as dots or a checkerboard design, and the density of the projected reference marks onto the surface could be varied depending on the severity of the curvature. For example, regions with high curvature (e.g., medial and lateral femoral condylar ridges) may benefit from a more dense pattern than regions with low curvature (e.g., femoral condylar groove).

The introduction of semi-automatic digitization of the targets directly from digital images including grid intersections and

Table 3 Specimens one $(4.50 \mathrm{~kg})$ and two $(4.95 \mathrm{~kg})$ : MDPG measurement precision for the bone and cartilage surfaces of the patella and femur. Mean standard deviation of the measured points ( $N=$ \# Pts) is given for the three coordinate directions. +MDPG reconstruction with five images.

\begin{tabular}{|c|c|c|c|c|c|c|c|c|c|}
\hline & \multicolumn{4}{|c|}{ Specimen 1} & \multicolumn{4}{|c|}{ Specimen 2} \\
\hline & & \multirow{2}{*}{$\stackrel{\#}{\text { Pis }}$} & \multicolumn{3}{|c|}{ Mean $\sigma(\mu \mathrm{m})$} & \multirow{2}{*}{$\begin{array}{c}\# \\
\text { Pis }\end{array}$} & \multicolumn{3}{|c|}{ Mean $\sigma(\mu m)$} \\
\hline & & & $\sigma_{x}$ & $\sigma_{y}$ & $\sigma_{2}$ & & $\sigma_{x}$ & $\sigma_{y}$ & $\sigma_{2}$ \\
\hline \multirow[t]{2}{*}{ Bone } & Patella & 37 & $10.3^{\dagger}$ & $10.4^{\dagger}$ & $17.8^{\dagger}$ & 78 & 8.4 & 10.4 & 12.6 \\
\hline & Femur & 81 & 15.6 & 15.2 & 27.4 & 111 & 14.1 & 14.2 & 21.1 \\
\hline \multirow[t]{2}{*}{ Cartulage } & Patella & 43 & 13.3 & 13.3 & 21.5 & 76 & 13.7 & 14.1 & 20.5 \\
\hline & Femur & 92 & 15.7 & 14.8 & 239 & 150 & 16.1 & 15.7 & 23.2 \\
\hline
\end{tabular}

posts using pattern fitting improves the quality of digitization. Using these methods, the operator does not need to concentrate on defining the target centre by hand and hence operator error is reduced. The operator indicates general regions near the target

Table 4 Specimen three (4.00 kg): MDPG measurement precision for the bone and cartilage surfaces of the patella and femur. Mean standard deviation of the measured points $(N=\# P t s)$ is given for the three coordlnate directions. Shaded rows indicate measurements that were made only on specimen three, and not on either specimens one or two. Femur Cond., Femur Epi., Femur Total indicate measurements on the fernoral condyle, femoral epicondyles, or summation of the Femur Cond. and Femur Epi. measurements, respectively.

\begin{tabular}{|c|c|c|c|c|c|}
\hline & \multirow{2}{*}{$\begin{array}{c}\# \\
\text { Pts }\end{array}$} & \multicolumn{3}{|c|}{ Mean $\sigma(\mu \mathrm{m})$} \\
\hline & & & $\sigma_{\mathrm{x}}$ & $\sigma_{y}$ & $\sigma_{\mathrm{z}}$ \\
\hline \multirow{4}{*}{ Bone } & Patella & 75 & 7.5 & 6.8 & 10.6 \\
\hline & Femur Cond & 174 & 79 & 77 & 104 \\
\hline & Futhur & 137 & 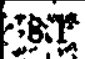 & 84 & $101 \%$ \\
\hline & Femiu Toral & 311 & 80 & -8.7 & 100 \\
\hline \multirow{4}{*}{ Cartilage } & Patella & 68 & 111 & 100 & 157 \\
\hline & Femur Cond & 99 & 107 & 120 & 157 \\
\hline & Fegman Epi & 118 & 14,5 & & 19 \\
\hline & & & & & $3 /$ \\
\hline
\end{tabular}


and the computer algorithms can "home in" on the precise target coordinates. However, although the algorithm is more objective at determining grid intersections than the traditional method of hand digitizing photographic prints, there is still an inherent element of subjectivity due to the user selectung where the horizontal or vertical lines are sampled to define the polynomials. Generally, the number of sampling points depends on the severity of the grid line curvature being sampled. Some element of subjectivity will always be present untıl a fully automatic algorithm can be devised. The use of digital imagery in photogrammetry may eventually lead to fully automatic reconstruction (Mitchell, 1995)

The self-calibrating bundle adjustment resulted in the precise determination of the interior camera parameters such as the focal length and principal points, and one of the possible seven distortion parameters, $a_{1}$, which models error in the CCD 1mage plane. While the statistical significance of the $a_{1}$ distortion parameter was confirmed using statistical tests (Förstner, 1985) from the self-calibrating bundle adjustments, there is a strong possibility that it is actually modeling a distortion other than for which it was intended. Fraser et al. (1995) showed that the $a_{1}$ parameter sometımes models an error signal in the camera lens (i.e., radial distortion or decenterıng distortion) rather than in the CCD image plane. We compared the $a_{1}$ value we determined to previous studies where, using the same camera, the $a_{1}$ value was determined with a high degree of consistency (Cosandier and Chapman, 1992; Lichti and Chapman. 1997) Because the comparison showed a different $a_{1}$ for this study than the previous studies, we concluded that it must be modelıng some component of radial or decentering distortion, rather than its intended image plane distortion.

Typically not all the distortion parameters can be resolved with statistical significance in a photogrammetric reconstruction. The number of parameters resolved depends on the quality of the lens used, and the design of the calıbration network. There are at least three possibilities why more than one distortion parameter could not be resolved in this setup. First, the distortions may not be present in the camera-lens setup ( I.e., distortionless). Second, the measurement precision of the twodimensional post locations in the photographs may not be sufficient to resolve distortion parameters. Third, the varıation of height of the calibratıon frame posts may be insufficient for resolving the distortion parameters The first possibility is unlikely, although it has been reported that the lens distortion can vary significantly with zoom lenses such as used in this study and that there exists a "sweet spot" in the lens where very little distortion occurs (Ray, 1994). If the zoom was coincidentally set at the sweet spot there may indeed have been very little distortion in the lens, how'ever the likelıhood of this seens low. The second possibılıty, that the two-dimensional post measurements in the photographs were insufficiently accurate to resolve the distortion parameters, is not likely beciluse the edge detection algorithm for the posts has been tested thoroughly in other applications of photogrammetry where more distortion parameters were successfully resolved (Cosandier and Chapman, 1992; Lichti and Chapman, 1997). The third possibılity, that the variation in height of the calibration frame posts was not sufficient (i.e., suboptimal calibration network design), is the most likely cause for the lack of resolution of distortion parameters. A characteristic of long focal length lenses is the inherent shallow depth of tield, which efficctively constrains the possibility for height viriation of the posts in the calibration frame. An aperture setting of $/ / 22$ was uned to maximize the depth of field in this study. An increased height variation of the calibration posts would improve the ability to determine distortion parameters; howeves, thıs woukl necessitate using a shorter focal length lens. A bulance between having precise surface measurements due to the long focal length lens and the maximizing of the depth of field for determunng distortion parameters must be made. The photogrammetric setup used for
Table 5 Measurement uncertainty $\left(2 \times \sigma_{z}\right)$ in the least favorable coordinate direction (height, $z$ ) is presented for MDPG and SPG (Ateshian et al., 1991, 1992). MDPG represents an improvement of the measurement uncertainty compared to SPG.

\begin{tabular}{|c|c|c|}
\hline \multirow{2}{*}{} & \multicolumn{2}{|c|}{ Measurement Uncertainty $\left(2 \times \sigma_{z}\right)$ in $\mu \mathrm{m}}$. \\
\cline { 2 - 3 } & MDPG & SPG \\
\hline \hline Plane & 6.2 & 25.0 \\
\hline Cylinder & 25.4 & 90.0 \\
\hline
\end{tabular}

this study resulted in an estimated measurement precision of the posts of $2 \mu \mathrm{m}$ (from the self-calibrating bundle adjustment) which is sufficient for measuring small joint surfaces.

The MDPG method represents an improvement over SPG for joint surface measurement. The measurement uncertainty with MDPG $\left(2 \times \sigma_{z}\right)$ for the cylinder and plane is presented for comparison with results from other studies (Ateshian et al., 1991, 1992) (Table 5). The improvement in accuracy of MDPG Is mainly due to the multistation image acquisition, which increases the redundancy of the imaging network and the $90 \mathrm{deg}$ convergence angle of the images, and the self-calibrating bundle adjustment, which makes optimal use of this imaging network by incorporating the nonlinear distortion model into the simultaneous solution. Although not all photogrammetric setups may necessitate modeling distortion parameters and utilizing multiple images (for example, when measurement precision is not paramount), the possibility for their addition to the adjustment make this calibration procedure more versatile than the SPG calibration. Fraser (1984) showed that the precision improvement of surface measurements using more than two images can be approximated by the following:

$$
\sigma \propto m^{-05}
$$

for large $m$ where:

$\sigma$ is the object point precision with $m$ images, and

$m$ is the number of spatially separated photographs.

Further improvements in measurement precision could possibly be attained by adding a thin layer of white paint to the cartilage and bone surfaces to improve grid contrast; however, it is not known how much the thickness of the paint would effect the overall surface measurement, and any treatment of the cartilage prior to measurement was avoided in general.

The multiple image information is used to determine the estimated reliability of each point on the surface individually so that poorly defined points (such as near the surface edge where the grid projection becomes increasingly tangential) can be discarded. This information can be used to estimate the overall photogrammetric reliability for every unique photogrammetric setup. Although individual point precision is possible with the SPG experimental setup (Atkinson, 1996), these estimations have not been reported. Rather, measurement accuracy with SPG is determined for each photogrammetric setup by performing a system accuracy test on a known solid cylinder. The determined system accuracy in one SPG setup (Ateshian et al., 1991) has been assumed to apply to surface measurements in subsequent similar SPG setups (e.g., Soslowsky et al., 1992). ln this study, it was demonstrated that photogrammetric measurement precision varies for each point on the joint surface, for each type of surface measured (steel, cartilage, or bone), and for every photogrammetric reconstruction. The variation of measurement precision can be observed in Tables 1,3 , and 4. For example, the poorest precision was obtained with the cartilage surfaces where the translucency of the cartilage combined with the curvature of the joints causes the grid lines to be 
diffuse. The precision was improved for the opaque subchondral bone surfaces; however, the curvature of the joints still resulted in distorted grid lines as they became tangent to the joint surface. The optimal precision estimates were obtained for the cylinder and plane surfaces because of the excellent contrast of the grid lines on the opaque surface and, in the case of the plane, complete lack of curvature.

The cartilage and subchondral bone surface measurement precision improved with each experiment as the MDPG technique was refined. The improvements were mainly due to building a new calibration frame and specimen ring, which improved the photogrammetric network. The specimen ring was designed to closely fit the specimen surface and thus maximized the size of the specimen in the photographs, which improved the photogrammetric reconstruction because the resolution of the images was increased. The calibration frame design was improved from its original design used with specimens one and two where the target posts were positioned around the perimeter of the photographs, to an improved design with its target posts isotropically distributed within the photographic field of view.

The question arises whether the excellent measurement precision of the photogrammetric reconstruction of the cat PF joint surfaces is a scaling effect solely due to the small size of the surfaces. Scaling likely has very minimal effects on the results. Lichti and Chapman (1997) showed that MDPG measurement precisions could be attained between $20 \mu \mathrm{m}$ and $40 \mu \mathrm{m}$ when measuring targets on a frame encompassing a volume of approximately $2 \mathrm{~m}^{3}$. The precision of the photogrammetric reconstruction is more likely to be affected by the precision at which the target coordinates are measured in a photograph, which is indirectly related to the resolution of the digital camera and the ability to digitize photographs accurately. Digital images facilitate more precise measures of the control post and grid targets using edge detection and pattern fitting algorithms than the previous method which involved manual digitization of photographic prints. The continual improvement of digital camera CCD resolution will help improve future reconstruction accuracies

The MDPG method is flexible for different joint sizes and for highly curved joints. The high number of images provides more fexibility for surfaces that "wrap" (i.e., the distal end of the femur) because camera positions can be located in optimal positions to view regions of the projected grid that become tangent to the joint surface. Also, the use of a zoom lens fitted with the digital camera is very flexible for measuring different sized surfaces. The use of a single camera to collect all the surface images reduces the numerical complexity of the selfcalibrating bundle adjustment and increases the reliability of the measurements because onty one set of interior and distortion parameters need to be determined. The cost of the MDPG system can be minimized because only one camera is necessary.

An added advantage to using the bundle adjustment method for surface reconstruction is that the results are not highly sensitive to calibration frame inaccuracies as is the case with the SPG method of re-section and intersection (Atkinson, 1996) reconstruction. The bundle adjustment incorporates so-called minimum constraints (Atkinson, 1996) where only seven parameters are constrained to define the object space (surface reconstruction). The calibration frame inaccuracies with the bundle adjustment method will result in an evenly distributed scaling error; however, these errors are expected to be small relative to the size of the reconstructed surface. Therefore, cost savings can be achieved over the SPG systems by constructing very simple and inexpensive control frames. This makes the system practical for laboratory use because a wide variety of frame sizes can be built to suit every photogrammetric setup.

The technique of MDPG is well established in the field of geomatics engineering for precise measurement of industrial machinery (Lichti, 1996). The present study was done in collaboration with geomatics engineers to adapt the technique of MDPG successfully to make precise measurements of joint sur- faces such as bone and cartilage, which could then be used to measure cartilage thickness and joint contact (e.g., Boyd, 1997). The field of digital photogrammetry is evolving rapidly and may eventually lead to fully automated methods for precise surface measurement. The successful implementation of MDPG is attributed mainly to the precise calibration procedure, the use of a redundant number of convergent digital photographs, edge detection, and pattern fitting algorithms, and a rigorous threedimensional reconstruction procedure, namely the bundle adjustment method. The successful application of this technique exceeded expectations for surface measurements of the small cat PF joint, and the technique offers an excellent non-contact method of acquiring precise surface geometry quickly, which can be useful for a variety of biomechanical applications.

\section{Acknowledgments}

This study was supported financially by Natural Science and Engineering Research Council of Canada and the Alberta Heritage Foundation for Medical Research. Significant technical assistance was provided by C. Sutherland, D. Young, B. Chaplin, Z. Canteenwalla, and A. Wuilbaut.

\section{References}

Atkinson, K. B , 1996, Close Range Photogrammetry and Machune Vision. Whittles Publishıng, Scotland, UK

Areshıan, G. A., Soslowsky, L. J., and Mow, V. C., 1991, "Quantitation of articular surface topology and cartilage thckness in knee jolnts usıng stereophotogrammetry," J. Btomechanics, Vol. 24, pp. 761-776.

Ateshıan, G. A., Rosenwasser, M P., and Mow, V. C., 1992, "Curvature characteristics and congruence of the thumb carpometacarpal joint differences between female and male joints," J. Bromechants, Vol. 25, pp. 59!-607.

Aleshian, G. A., Kwak, S. D., Soslowsky, L J, and Mow, V C.. 1994, "A stereophotogrammetric method for determining in situ contact areas in diarthrodial joints, and a comparison with other methods." I Biomechanics, Vol. 27, No. 1, pp. $111-124$

Blankevoon, L., Huiskes, L., and de Lange, A., 1988. "The envelope of passive knee joint motion, " J Biomechanics, Vol. 2I, pp. 705-720

Boyd, S. K, 1997, "A 3D In-Situ Model for Patellofemoral Joint Contact Analysis in the Normal and Anterior Cruciate Ligament Deficient Knee," MSc. Thesis, University of Calgary Press, Calgary, AB.

Boyd, S. K, and Ronsky, J. L, 1998. 'Instantaneous Moment Arm Determination of the Cat Knee Jomt," J. Biomecharics. Vol. 31, No 3, pp. 279-283.

Boyd, S. K.. Ronsky, J. L., Lichti, D. D., Salkauskas, K., and Chapman, M. A., 1997, "Quantıfication of Articular Cartilage Thickness of the Cat Patellofemoral Joint Wuth Multı-Station Digital Photogrammetry and Thın-Plate Spline Surface Interpolation." Proc Bloengineering Conference ASME, Vol. 35. pp. 43-44.

Bray. R C, Shnve. N. G, Frank, C. B.. and Chimich, D. D. 1992, "The early effects of joint immobilization on the medial collateral Jigament healing in an ACL-deficient knee: $\mathbf{A}$ gross anatomic and biomechanical investigation in the adult rabbut model," J Orthop. Res., Vol. 10, pp. 157-166.

Cosandier, D., and Chapman, M. A., 1992. "High precision target location for Industral metrology," SPIE Videonetrics, Vol. 1820, pp. 111-122.

Förstner, W. 1985, "The reliability of block triangulation," Photogrammetric Engineering and Remote Sensing, Vol. 51, No. 6, pp .1137-1149.

Fraser, C. S , 1980, "Multiple focal setting self-calibration of close-range metric cameras," Photogrammetric Engineering and Remote Sensing, Vol. 46, No. 9. pp 116$]-117]$.

Fraser. C. S, 1982. "Optimızation of precision in close-range photogramme. try." Pholngrammetric Engineering and Remole Sensing. Vol 48, No. 4, pp. $561-570$.

Fraser, C. S . 1984, "Network design considerations for non-topographic photogrammetry," Photogrammetric Engineering and Remote Sensing. Vol. 50, No. 8, pp. $1115-1126$

Fraser, C. S., Shonts. M. R, and Gancı, G. 1995. "Multi-sensor system selfcalibration," Proc. Videometrics IV. Vol. 2598, pp. 2-18

Ghosh, S. K. 1983, "A close-range photogrammetric system for 3-D measurements and perspective diagramming in biomechanics." J. Biomechanics, Vol. 16, pp. 667-674

Granshaw, S. I., 1980, "Bundle adjusiment methods in engineerıng photogrammetry," Photogrammetre Record. Vol. 10. No. 56, pp. 18I-207

Herzog, W., Adams, M. E.. Matyas, J. R., and Brooks, J. G., 1993. "Hindlimb loadıng, morphology and biochemıstry of articular cartilage in the ACL-deficsent cat knee," Ostevarrhritis and Carilage. Vol. 1, pp. 243-25l.

Huiskes, R., Kremers, J, de Lange, A.. Woltring. H. J., Selvik, G., and van Rens, J. G., 1985, "Analytical Siereophotogratnmetnc Delermınarion of ThreeDimensional Knee-Joint Geometry," J. Biomechanics, Vol. 18, No. 8, pp. 559570 .

Korvick, D L., Pijanowski, G. J., and Schaeffer, D. J., 1994, "Three-dimensional kinematics of the intact and cranial cnuciate hgament-deficient stifle of dogs." J. Biomechanics, Vol. 27, pp 77-87 
Kwak, S. D. Colman, W. W., Ateshıan. G A. Grelsamer, R P. Henry. J. H., and Mow, $\vee \mathrm{C}, 1997$. "Anatomy of the human patellofemoral joint articular cartlage: surface curvature analysis," J. Orthop Res. Vol. 15. pp 468-472

Lichti, D. D. 1996, "Constraned finite element merhod self-calibration." M Sc. Thesis, Unjyersity of Calgary Press, Calgary, AB

Lichti, D D, and Chapman, M A , 1997, "Constrained FEM self-calihration,' Phorogrammerrc Engineering \& Remote Sensing, Vol 63. No. 9, pp. 11111119.

Lichti, D D , Chapman, M A. Boyd. S. K, and Ronsky, J. L, 1997, "Digital photogrammetric measurement of knee joint surfaces." Photogrammetry and Remote Sensing, Vol. 3. pp. 283-292.

Mitchell, H L., 1995, "Applications of distal photogrammetry to medical iлvestigations." ISPRS Journal of Photogrammetry and Remote Sensing, Yol. 50. No. 3, Pp $27-36$

Pond, M J . and Nuk1, G 1973, 'Experımentally-snduced osteoarthritus in the dog," Ann. Rheum. Dis., Vol 32, pp. 387-388

Ray, S. F.. 1994. Applied Photographic Opnics. 2nd ed., Focal Press, Oxford, UK.

Ronsky, J. L. J994, "In-vivo patellofemoril jolnt contact," PhD Thesis, UnIversity of Calgary Press, Calgary, AB

Ronsky, J. L, Herzog, W, Brown, T D, Pedersen, D R \& J E S. and

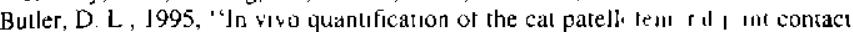
stresses and areas, I Biomechancs. Vol 28, No. 8, pp. of
Ronsky, J. L, Boyd, S K, Lichti, D D., Chapman, M. A., and Salkauskas, K , 1997, "Precise Measurement of Articular Cartilage Surface. Comparison of MultıStation Digital Photogrammerry With 3D Digitizatıon," Proc Btoenginecring Conference ASME, Vol. 35, pp. 39-40

Scherrer. P K, and Hillberry. B. M., 1979, "Pıece-wise maihematical representation of articular surfaces," J. Binmechanics, $V_{0}$ l2, pp $30 \mathrm{l}-31 \mathrm{I}$.

Seedhom, B B . Longton, E. B.. Wright, V., and Dowson, D , 1972. "Dimensions of the knee." Ann. Rheum. Dis. Vol. 31, pp 54-58.

Shıba, R., Sorbı, C., Siu, D. W., Bryant, J.T., Cooke, D V. and Wevers, H W , 1988, "Geometry of the humeroulnar join,," J Orrhop. Res, Vol. 6, pp $897-906$

Soslow'sky, L. J., Flatow, E. L, Bigliant. L U, Pawluk, R J.. Ateshtan. G. A., and Mow, V. C., 1992, "Quantutation of In Situ Contacl Aiteis at the Glenohumeral Joint: A Biomechanical Study," J Orihop Res, vol 10. pp 524-534

Tabatabai, X X, and Mitchell. Y Y., 1984, "Edge location to subpixel values in digital imagery," IEEE Transactions un Pattern Analysis and Machine Intelligence, $V_{0}$ 6. No. 2, pp. $188-201$.

Wismans, J, Veldpaus, F., Janssen, J., Huson, A.. and Siruben, P., 1980, "A inree-dimensional mathematical moxlel of the knee-joint," J Biomechamics, Vol. 13, pp 667-685

Wijk, Mi C. vın, 1980, "Mordc contourography-un accuracy analysis." J. Biomechanics, Vol. 13, No 7.pp 605-613

The World's Most Active Engineering Site - The Worlo's Most Trusted Engineering Organization Periodicals on Fouturg...

- Tables of Contents for most recent Issues of Transactions Journals

ASMENET

- Full text of Heat Transfer - Recent Contents online

- Online Publications Catalog

- Timely, accurate information about ASME's publications via TechAlert ASME's new e-mail IIstserv

(http://www.asme.org)

- Links Junction - links to US National Labs, libraries, engineering organizations

\section{Transactions of the ASME}

ASME Transactions Journals are world-class repositorles of the flnest engineering literature from top researchers in the!r fields. Each refereed journal contains articles designed to keep readers abreast of current theory and practice and serve as a permanent technical record of engineering research and development. 